Jerzy KREMKY.

\title{
Argynnis amathusia Esp. f. bialovieżensis Gieysztor.
}

Dr. M. Gieysztor hat in seiner Notiz „Sprawozdanie $z$ pracy nad fauną motyli większych (Macrolepidoptera) Puszczy Białowieskiej“ (Białowieża, Heft 2, Warszawa, 1923, S. 119139) eine Aberration von Argynnis amathusia Es p. ab. bialovieżensis ab. nov. beschrieben. Die Exemplare dieser Form hat Dr. Gieysztor, zusammen mit seinem sonstigen lepidopterologischen Material aus dem Urwalde von Białowieża, an das Polnische Zoologische Staatsmuseum abgegeben. Auf Grund einer eingehenden Durchsicht der betreffenden Literatur, sowie des Vergleichs der genannten Exemplare mit den Materialien, welche sich im Polnischen Zoologischen Staatsmuseum in Warschau und im Naturhistorischen Staatsmuseum in Wien befinden, ferner mit Exemplaren, die Herr Prof. N. J. Kusnezov aus verschiedenen Gegenden Russlands und Herr Mag. W. Peters e n aus Estland zur Untersuchung liebenswürdig zusandten, komme ich zur Überzeugung, dass die durch Dr. Gi eys z tor beschriebene Form von allen bisher bekannten abweichend ist und nicht als eine individuelle Aberration, sondern als eine für Białowieża charakteristische Lokalform angesehen werden soll.

Wie überhaupt bei der hier in Frage kommenden Art die Unterseite der Hinterflügel äusserlich am meisten bezeichnend erscheint, so sind auch für die f. bialovieżensis Gieysztor die wesentlichsten unterscheidenen Merkmale daselbst zu suchen. 
Die Flügelspannung der mir vorliegenden Exemplare schwankt von 42 bis $46 \mathrm{~mm}$. Die oben dunkel gefärbten Flügel erinnern an die alpine Form titania Hb. (serena Fruhst.). An der Unterseite der beiden Flügelpaare sind die dreieckigen Zacken am Aussenrand viel kürzer als bei anderen europäischen Formen, was an die sibirische Form sibirica Stgr. erinnert. Der rhomboidale Fleck, welcher in der Mittelbinde der Hinterflügel, zwischen der Subcostal- und der Radialader liegt, ist breit und berührt die genannten Adern, was wiederum an die Verhältnisse bei den in der Sammlung des Polnischen Zoologischen Staatsmuseums befindlichen, ostsibirschen Exemplaren erinnert.

Die ganze Binde ist einfarbig, gelb, wodurch sich f. bialovieżensis $\mathrm{G}$ ieys $\mathrm{z}$. der osteuropäischen Form bivina $\mathrm{F} \mathrm{r}$ u h $\mathrm{t}$. aus Saratov nähert. Übrigens unterscheidet sie sich jedoch sehr stark von dieser letzteren, welche kleiner, oben heller, dafür aber von unten noch dunkler als. f. titania $\mathrm{Hb}$. ist. Bei der f. bialovieżensis Gieyszt. ist die Grundfarbe der Hinterflügel von unten ausgesprochen gelb, weshalb sich die Mittelbinde von dieser wenig abhebt. Nur bei einigen Exemplaren erscheint die Mittelbinde längst den Adern sehr schwach rot angestreut. Bei diesen Exemplaren ist auch die Grundfarbe der Flügel etwas stärker rötlich angelaufen. Die weisse Farbe fehlt hier vollständig, die silberne ist nur bei einigen Exemplaren sehr spärlich bemerkbar, wodurch sich die Form von der f. amathusia Esp. aus der Umgegend von Leningrad (die Exemplare aus Estland erinnern sehr an diese Form) und von der f. titania $\mathrm{Hb}$. aus den Alpen unterscheidet. Im ganzen bieten die Hinterflügel der f. bialovieżensis $\mathrm{G}$ i e ys z t. von unten ein ausgesprochen helles Aussehen.

Die Formen jugurtha Fruhst. (Bayern), bosna Fruhst. (Bosnien), dinara Fruhst. (Herzogovina), blachieri Fruhst. (Tessin), pralignana Fruhst. (Savoyen), halesa Fruhst. (Pyrenäen), transsilvanica Titlscher (Siebenbürgen) und altaica Seitz (Altai) weichen von der f. bialovieżensis Gieyszt. ebenfalls ab.

In der Sammlung des Polnischen Zoologischen Staatsmuseums finden sich 6 Exemplare der f. bialovieżensis Gieyszt., sämtliche aus dem Urwald von Białowieża, erbeutet am $28 \mathrm{VI}$, 1 und 6 VII 1922, sowie am 8 und 13 VII 1923 (leg. Dr. M. Giey- 
sztor). Ein siebentes Exemplar hat Herr Dr. Gieysztor seiner Zeit dem Regionalmuseum in Białowieża überlassen. Die typische Form ist aus Białowieża nicht bekannt.

\section{LITERATURVERZEICHNIS.}

1. Fruhstorfer H. Neue Argynnis und Melitaea. Internationale Entomologische Zeitschrift, I, 1907-1908, Guben, S. 310.

2. Fruhstorfer H. Neue palaearktische Rhopaloceren Ibidem, II, 19081909 , SS. $214-215$.

3. Fruhstorfer H. Neue palaearktische Rhopaloceren Ibidem, III, 19091910 , SS. $20-21$.

4. G a e de M. In S e itz. Die Grossschmetterlinge der Erde. Fauna Palaearctica. Supplement. I, Stuttgart, 1930, S. 221.

5. Gie ysztor M. Sprawozdanie z pracy nad fauną motyli większych (Macrolepidoptera) Puszczy Białowieskiej. Białowieża, zesz. 2, Warszawa, SS. $119-139,1923$.

6. Osthelder L. Die Schmetterlinge Südbayerns und der angrenzenden nördlichen Kalkalpen. I Teil, 1 Heft, München, 1925. S. 98,

7. Pet er se n W. Lepidopteren-Fauna von Estland (Eesti). Teil I, Tallinn, 1924, S. 105.

8. Seitz A Die Grossschmetterlinge der Erde. I, Stuttgart, 1908, S. 232.

STRESZCZENIE.

Autor podaje, że opisana w swoim czasie Argynnis amathusia Esp. ab. bialovieżensis Gieyszt or nie jest odmianą indywidualną, lecz formą lokalną, charakterystyczną dla Puszczy Białowieskiej. 